\title{
Two types of adjacent dimer layers in the low-temperature phase of $\mathrm{BaCuSi}_{2} \mathrm{O}_{6}$
}

\author{
D. V. Sheptyakov* and V. Yu. Pomjakushin \\ Laboratory for Neutron Scattering, Paul Scherrer Institut, CH-5232 Villigen PSI, Switzerland \\ R. Stern ${ }^{\dagger}$ and I. Heinmaa \\ National Institute of Chemical Physics and Biophysics, Tallinn 12618, Estonia
}

H. Nakamura and T. Kimura

Division of Materials Physics, Graduate School of Engineering Science, Osaka University, Toyonaka, Osaka 560-8531, Japan

(Received 21 May 2012; published 30 July 2012)

\begin{abstract}
The low-temperature crystal structure of $\mathrm{BaCuSi}_{2} \mathrm{O}_{6}$ has been investigated with high-resolution synchrotron $\mathrm{x}$-ray and neutron powder diffraction techniques and has been found to be on average (ignoring the incommensurate modulation) orthorhombic, with the most probable space group I bam. The $\mathrm{Cu}-\mathrm{Cu}$ dimers in this material are forming two types of layers with distinctly different interatomic distances. Subtle changes also modify the partially frustrated interlayer $\mathrm{Cu}-\mathrm{Cu}$ exchange paths. The present results corroborate the interpretation of low-temperature nuclear magnetic resonance and inelastic neutron scattering data in terms of distinct dimer layers.
\end{abstract}

DOI: 10.1103/PhysRevB.86.014433

PACS number(s): 61.05.C-, 61.05.fm, 75.50.-y, 64.60.-i

\section{INTRODUCTION}

Intense studies of field-induced quantum phase transitions (QPTs) in various magnetic insulators continue to enrich our knowledge of the possible quantum ground states of matter. ${ }^{1-4}$ Structurally dimerized quantum spin systems play a leading role in these studies; the classes of field-induced QPTs known to date in these systems are well summarized in Refs. 5 and 6. For magnetic interactions with weak or no frustration, the kinetic energy of the triplet quasiparticles in such systems is dominant and the ordered, or Bose-Einstein condensed (BEC), ground state is uniform at $H>H_{c}$, as, e.g., in $\mathrm{TlCuCl}_{3}{ }^{4,7}$ In $\mathrm{SrCu}_{2}\left(\mathrm{BO}_{3}\right)_{2}{ }^{4,8,9}$ triplet hopping is suppressed by geometrical frustration and the repulsion causes the condensed triplets to form a superlattice with the appearance of magnetization plateaus. To better distinguish between the various scenarios and to determine accurately the relevant exchange couplings adequate knowledge of crystal structures and symmetries is of outmost relevance.

The interest in $\mathrm{BaCuSi}_{2} \mathrm{O}_{6}$ is motivated by its extraordinary phase diagram with field-induced BEC. ${ }^{6,10,11}$ Being a quantum paramagnet at zero magnetic field down to the lowest temperatures, the system displays a QPT into a magnetically ordered state at the critical value of magnetic field of $\sim 23.5 \mathrm{~T}^{10,11}$ At $\sim 610 \mathrm{~K}$, the crystal structure of $\mathrm{BaCuSi}_{2} \mathrm{O}_{6}$ undergoes a phase transition from the high-temperature (HT) phase with space group $I 4 / \mathrm{mmm}$ and lattice parameters $a_{h t} \sim 7.11 \AA$, $c_{h t} \sim 11.2 \AA$ to its room-temperature (RT) phase with a space group $I 4_{1} /$ acd and lattice parameters $a_{r t} \sim \sqrt{2} a_{h t} \sim$ $10 \AA, c_{r t} \sim 2 c_{h t} \sim 22.5 \AA{ }^{12}$ In the essentially layered RT tetragonal crystal structure of $\mathrm{BaCuSi}_{2} \mathrm{O}_{6},{ }^{12,13}$ the copper silicate $\mathrm{Cu}_{2}\left(\mathrm{SiO}_{3}\right)_{4}$ layers are separated by the intermediate layers of $\mathrm{Ba}$ atoms (illustration in Fig. 1). The $\mathrm{Cu}-\mathrm{Cu}$ dimers with an interatomic distance of $\sim 2.75 \AA$ are well separated from each other in the structure, since the interdimer $\mathrm{Cu}-\mathrm{Cu}$ distances within the layer $(\sim 7 \AA)$ and the $\mathrm{Cu}-\mathrm{Cu}$ distances between the dimers in adjacent layers $(\sim 5.75 \AA)$ are much larger. Thus, to a great extent, the ground state of the system is determined by the $\mathrm{Cu}-\mathrm{Cu}$ interactions within the dimers. ${ }^{14,15}$
At zero magnetic field, the $(S=1 / 2) \mathrm{Cu}^{2+}$ ions are paired antiferromagnetically, while the degenerate excited triplet states with $S=1$ are gapped; ${ }^{14}$ thus the compound possesses an essentially singlet ground state with $S=0$ at $H=0$. Upon application of a magnetic field, the excited triplet states are Zeeman split with the energy scale of the splitting being proportional to magnetic field. At a critical magnetic field value of $H_{c} \sim 23.5 \mathrm{~T}$, the energy of the lowest triplet state with $S_{z}=+1$ becomes lower than that of a singlet state with $S=0$, and a magnetic field-induced BEC of the excitations occurs. The population of the bosons (measured as the magnetization of the sample) may be precisely tuned by the magnetic field. Another intriguing phenomenon observed in $\mathrm{BaCuSi}_{2} \mathrm{O}_{6}$ next to this QPT is dimensional crossover around $1 \mathrm{~K}$ from a $3 \mathrm{D}$ into a $2 \mathrm{D}$ regime with lowering temperature. ${ }^{11,16}$ To explain this feature, perfect frustration between adjacent $\mathrm{Cu}_{2}\left(\mathrm{SiO}_{3}\right)_{4}$ layers was assumed. ${ }^{11,16,17}$ Alternatively, the hypothesis has emerged that a structural modulation along the $c$ axis s, $^{5,19}$ combined with nonperfect frustration is the sole reason for the lowered dimensionality. ${ }^{20,21}$ As a unifying theory, a possibility has been proposed ${ }^{22}$ that the combined effect of the structural modulation along the $c$ axis and the nearly perfect interlayer frustration is a reason for the observed dimensional crossover.

In attempts to build a theory of this QPT, the compound was originally assumed to possess the same square motif of the $\mathrm{Cu}$ $\mathrm{Cu}$ dimers at low temperatures as at room temperature ${ }^{10,11,23}$ resulting in three relevant coupling constants entering the spin Hamiltonian: the intradimer, interdimer, and interlayer couplings.

However, high-resolution inelastic neutron scattering (INS) data were clearly at odds with this picture. ${ }^{5}$ Furthermore, a first-order structural phase transition at $\sim 100 \mathrm{~K}$ has been discovered by Stern et al. ${ }^{19}$ and reported by Samulon et al. ${ }^{24}$ which necessarily states that the true spin Hamiltonian of the system is more complex than originally thought. The method of single crystal diffraction used in Ref. 24 confirmed the transition into an orthorhombic (or weakly monoclinic) structure. Moreover, it allowed for observation of satellite 


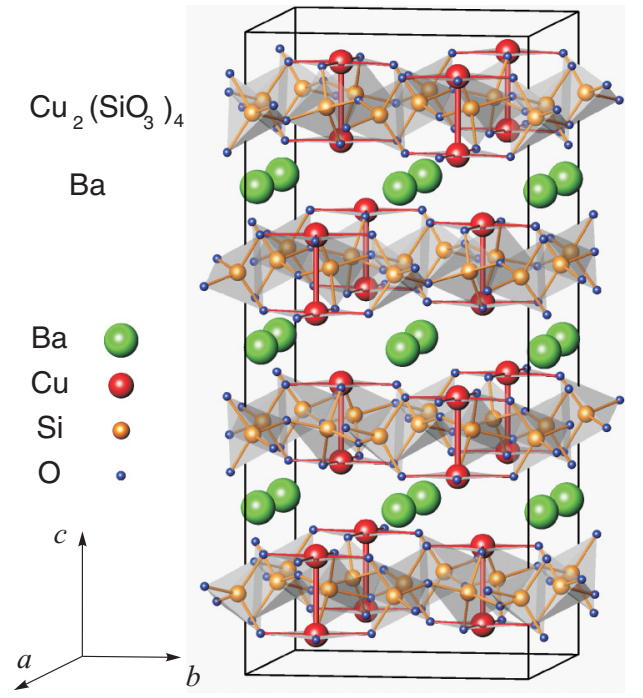

FIG. 1. (Color online) General view of the room temperature tetragonal crystal structure of $\mathrm{BaCuSi}_{2} \mathrm{O}_{6}$. Copper silicate $\mathrm{Cu}_{2}\left(\mathrm{SiO}_{3}\right)_{4}$ layers are interleaved with the layers of $\mathrm{Ba}$ atoms. The drawing is based on the crystal structure parameters refined from neutron powder diffraction data collected at $T=130 \mathrm{~K}$.

peaks indicating an incommensurate modulation in the lowtemperature (LT) phase of $\mathrm{BaCuSi}_{2} \mathrm{O}_{6}$. Here we use in contrast a combination of high-resolution x-ray and neutron powder diffraction techniques in order to determine the average crystal structure of the LT phase of $\mathrm{BaCuSi}_{2} \mathrm{O}_{6}$.

\section{EXPERIMENTAL}

\section{A. Synthesis}

Polycrystalline samples of $\mathrm{BaCuSi}_{2} \mathrm{O}_{6}$ were prepared by solid-state reaction. Powders of $\mathrm{BaCO}_{3}, \mathrm{CuO}$, and $\mathrm{SiO}_{2}$ were weighted to the prescribed ratios, mixed, and well ground. The mixture was calcined at $900{ }^{\circ} \mathrm{C}$ in air for $20 \mathrm{~h}$. The resulting powders were pulverized, isostatically pressed into a rod shape ( $\sim 5 \mathrm{~mm}$ diameter, $\sim 50 \mathrm{~mm}$ length), and sintered again at $1010{ }^{\circ} \mathrm{C}$ in air for $20 \mathrm{~h}$.

\section{B. Powder diffraction experiments}

The powder diffraction experiments have been carried out with two high-resolution diffractometers: the Powder Diffraction station of the Materials Sciences Beamline (MS-PD) at the Swiss Light Source, ${ }^{25}$ and the high-resolution powder neutron diffractometer HRPT ${ }^{26}$ at the spallation neutron source SINQ, both at Paul Scherrer Institute in Villigen.

At the MS-PD station, the synchrotron x-ray diffraction data were collected on a powder sample enclosed in a capillary of a $0.3 \mathrm{~mm}$ diameter, which was placed in a Janis flow-type cryostat. The Microstrip Mythen-II detector was used, which allowed for high counting rates while maintaining the high resolution which was essentially sample-conditioned. The typical counts of $\sim 2 \times 10^{5}$ in the strongest peaks were achieved within $\sim 1$ minute. On cooling, the diffraction patterns were collected with $1 \mathrm{~K}$ steps in the temperature range from 154 to $64 \mathrm{~K}$, and with $2 \mathrm{~K}$ steps in the temperature range from 64 to $12 \mathrm{~K}$. After collecting the lowest temperature data set at $4 \mathrm{~K}$, the data were collected on heating the sample in the temperature range from 5 to $123 \mathrm{~K}$ with typically $1 \ldots 2 \mathrm{~K}$ steps and from 128 to $178 \mathrm{~K}$ with $5 \mathrm{~K}$ steps, in order to observe the transition both on cooling and on heating and to characterize the hysteresis thereof.

At HRPT, a bigger amount ( $\sim 1 \mathrm{~g})$ of the very same powder sample of $\mathrm{BaCuSi}_{2} \mathrm{O}_{6}$ was enclosed into a vanadium can of a $6 \mathrm{~mm}$ diameter, and the data were collected using a close-cycle refrigerator at $130 \mathrm{~K}$ (well above the transition on cooling) and at $13 \mathrm{~K}$ (far below the transition) in the instrument setup with the highest resolution. The ultimate resolution was needed because the orthorhombic splitting of the peaks of the parent tetragonal RT structure characterized by an orthorhombicity parameter defined as $2\left|a_{\text {orth }}-b_{\text {orth }}\right| /\left(a_{\text {orth }}+b_{\text {orth }}\right)$ amounts only to $\sim 1.7 \times 10^{-3}$.

The data of the neutron diffraction experiment were used for the structure determination, and for the precise refinement of the structural parameters at low temperature, while the synchrotron $\mathrm{x}$-ray powder diffraction data have mainly been used for indexing, space group selection, and refinement of the temperature dependence of the main structural parameters, as well as for identifying all the impurity phases present. The following impurities have been determined in the powder sample: $\mathrm{BaCu}_{2} \mathrm{Si}_{2} \mathrm{O}_{7}$ ( 4.4\% wt.), $\mathrm{BaCuSi}_{4} \mathrm{O}_{10}$ ( 1.3\% wt.), $\mathrm{Ba}_{4} \mathrm{Si}_{6} \mathrm{O}_{16}(\sim 1.1 \%$ wt. $), \mathrm{BaSiO}_{3}(\sim 0.3 \%$ wt. $)$, and $\mathrm{Cu}_{2} \mathrm{O}$ ( $\sim 0.1 \%$ wt.). Approximately $93 \%$ wt. of the powder sample is the main $\mathrm{BaCuSi}_{2} \mathrm{O}_{6}$ phase. While treating the neutron data with $\sim 5000$ counts in the strongest peak, the only relevant impurity which was definitely seen in the diffraction pattern and correspondingly input into the refinements was $\mathrm{BaCu}_{2} \mathrm{Si}_{2} \mathrm{O}_{7}$ ( $\sim 4.4 \%$ wt.); the others were undetectable, and thus were not considered.

The RT crystal structure model (Ref. 12) has been unambiguously confirmed by the Rietveld refinements based on both synchrotron $\mathrm{x}$-ray and neutron data above the phase transition temperature. We do not focus on this issue here, but rather concentrate on the LT structure.

\section{RESULTS}

\section{A. Low-temperature crystal structure of $\mathrm{BaCuSi}_{2} \mathrm{O}_{6}$}

The LT structural phase transition in $\mathrm{BaCuSi}_{2} \mathrm{O}_{6}$ is clearly seen in both neutron and x-ray diffraction patterns, and is most evident in the temperature-dependent X-ray data. Illustrations of the transition are given in Figs. 2 and 3. While all the peaks get a little narrower when cooling below the transition, we do not observe any of them to broaden. Instead, some remain single peaks, and some are splitting. The peak $(2,2,12)$ at $\sim 17.35^{\circ}$ remains single, while the $(6,0,4)$ and $(6,2,0)$ at $\sim 17.9^{\circ}$ and $\sim 18.15^{\circ}$ become split into the orthorhombic pairs $(0,6,4) /(6,0,4)$ and $(2,6,0) /(6,2,0)$, correspondingly. The peak $(8,0,0)$ at $\sim 23^{\circ}$ splits into a $(0,8,0) /(8,0,0)$ doublet, and the strong $(6,4,8)$ at $\sim 23.1^{\circ}$ transforms into a pair of $(4,6,8) /(6,4,8)$ which are not resolved, since the amplitudes of their scattering vectors are too close to each other. The backward phase transition on heating manifests itself (see Fig. 3) as a recovery of the diffraction pattern to that of the RT crystal structure, yet it occurs at a higher temperature. 

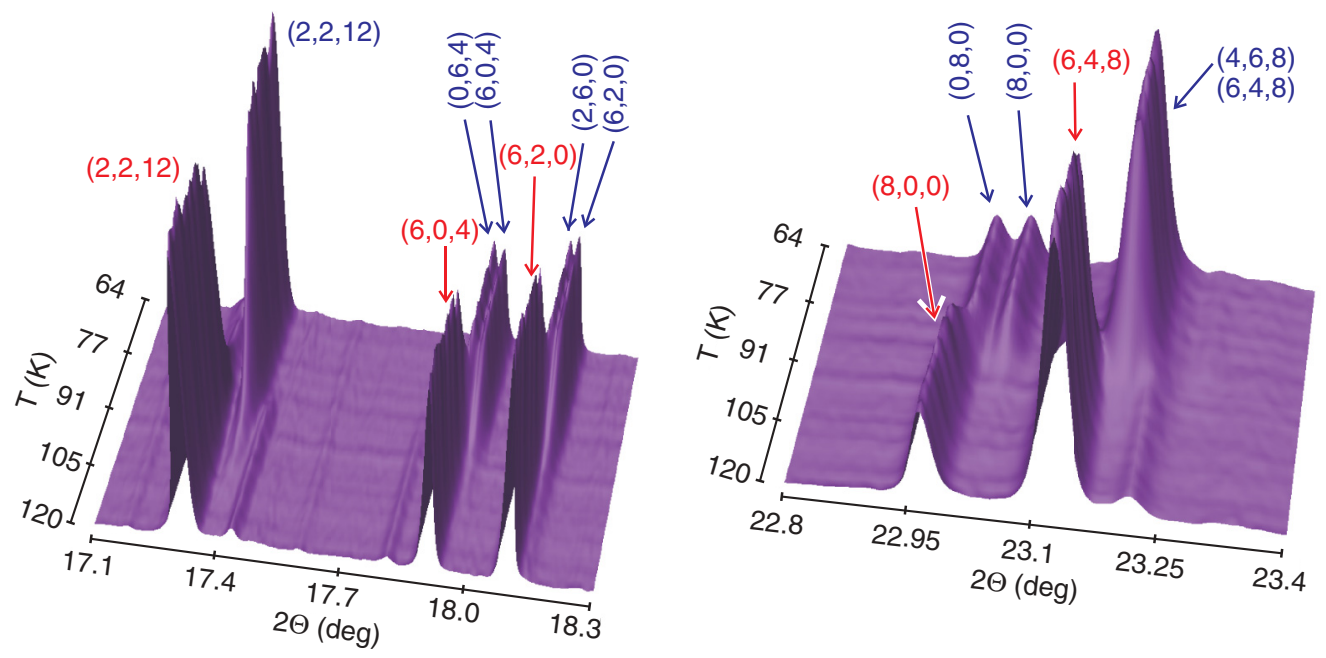

FIG. 2. (Color online) Selected ranges of the synchrotron $\mathrm{x}$-ray powder diffraction patterns of $\mathrm{BaCuSi}_{2} \mathrm{O}_{6}$ taken with $1 \mathrm{~K}$ steps on cooling from 120 to $64 \mathrm{~K}$. Obvious are the jump in the unit cell volume at the transition at $\sim 89.7 \mathrm{~K}$ (all the main peaks are shifted to higher $2 \Theta$ angles) and the splitting of the diffraction peaks with $h \neq k$.

The LT synchrotron x-ray diffraction pattern of $\mathrm{BaCuSi}_{2} \mathrm{O}_{6}$ showing clear splitting of the diffraction peaks $(h k l)$ with $h \neq k$ of the parent room-temperature tetragonal crystal structure (space group $I 4_{1} /$ acd ) has been indexed on the orthorhombic unit cell with lattice constants (at $T=4 \mathrm{~K}$ ) $\{a, b, c\}=\{9.951 \AA, 9.968 \AA, 22.239 \AA$. From the systematic absences it has been found that the most probable space group is body-centered, and all the relevant possibilities have been checked for the structure determination. In fact we have performed the structure determination for all space groups and combinations of $(a, b, c)$, which did account for all diffraction

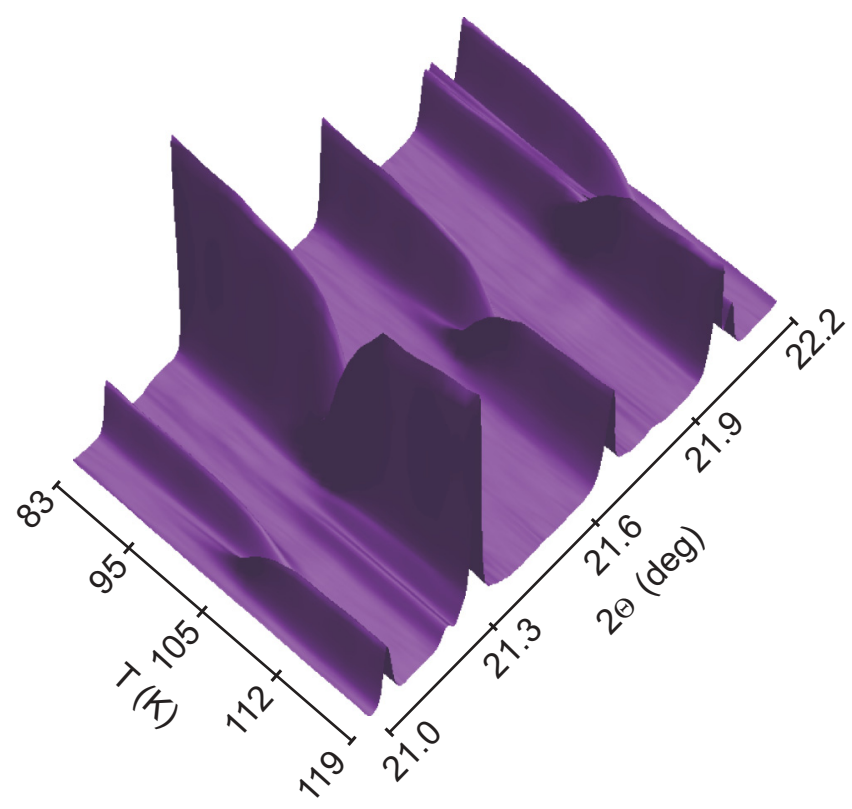

FIG. 3. (Color online) Selected range of the synchrotron $x$-ray powder diffraction patterns of $\mathrm{BaCuSi}_{2} \mathrm{O}_{6}$ taken with $2 \mathrm{~K}$ steps on heating from 83 to $97 \mathrm{~K}$ and with $1 \mathrm{~K}$ steps on heating from 99 to $119 \mathrm{~K}$ (note the nonuniform scale on the temperature axis). One clearly sees the structural phase transition occurring (on heating) at $\sim 107.2 \mathrm{~K}$. peaks observed, although for many of these some of the calculated peaks were actually absent in the experimental pattern. Having done so, we may exclude any chance for having missed the true solution. The structure determinations have been carried out on a neutron diffraction data set taken at $T=$ $13 \mathrm{~K}$, either with the program $\mathrm{FOX}^{27}$ or by direct symmetry reduction in cases where it was possible and straightforward. All the models have been checked with extensive Rietveld refinements (done with the program FULLPROF ${ }^{28}$ ) of their parameters from both the synchrotron $\mathrm{x}$-ray and neutron powder diffraction patterns.

While going from higher to lower symmetries in the orthorhombic syngony, already the space group Ibca (No. 73), which is a direct subgroup of the RT structure space group

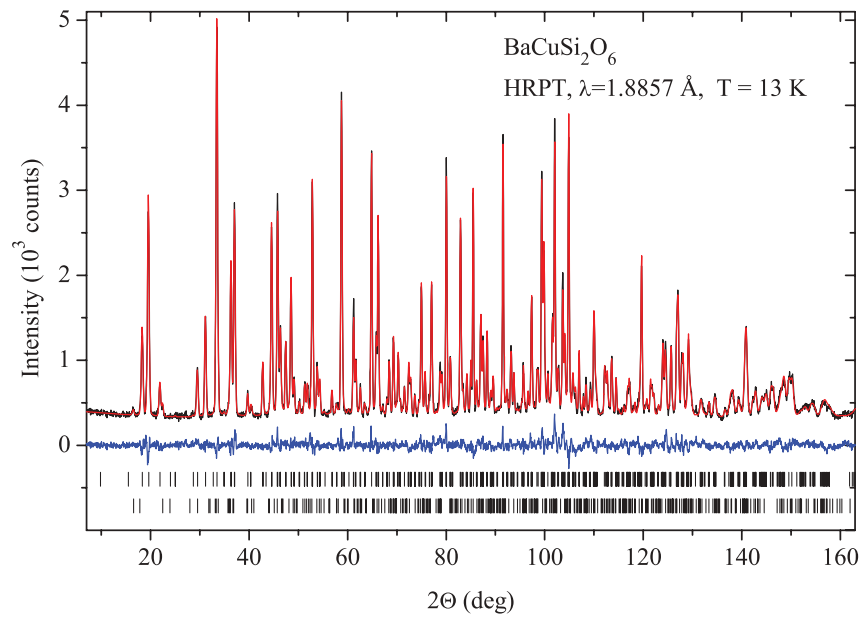

FIG. 4. (Color online) Rietveld refinement of the crystal structure parameters of the low-temperature (LT) phase of $\mathrm{BaCuSi}_{2} \mathrm{O}_{6}$ from the neutron powder diffraction data. The observed intensity, calculated profile, and difference curve are shown. The rows of ticks at the bottom correspond to the calculated diffraction peak positions of the phases (from top to bottom): $\mathrm{BaCuSi}_{2} \mathrm{O}_{6}$-orthorhombic LT structure, $\mathrm{BaCu}_{2} \mathrm{Si}_{2} \mathrm{O}_{7}$ impurity ( $\sim 4 \%$ wt.). 
TABLE I. The refined crystal structure parameters of the LT phase of $\mathrm{BaCuSi}_{2} \mathrm{O}_{6}$. Results obtained from the refinement done on neutron powder diffraction data collected at $13 \mathrm{~K}$ with the wavelength $\lambda=1.8857 \AA$. Space group I $\operatorname{bam}$ (No. $72, Z=16$ ). Isotropic temperature factors were refined with a constraint to equality for $\mathrm{Cu}$ atoms, for $\mathrm{Si}$ atoms, for the $\mathrm{O} 1 \cdots \mathrm{O} 4$ atoms ("apical" ones of the $\mathrm{SiO}_{4}$ tetrahedra, also coordinating $\mathrm{Cu}$ in the $\mathrm{CuO}_{4}$ squares), and for the $\mathrm{O} 5 \cdots \mathrm{O} 7$ atoms (directly bridging the $\mathrm{Si}$ atoms, i.e., located at exactly or roughly the same $z$ values as $\mathrm{Si}$ ). Atoms are located in the following crystallographic positions: $\mathrm{Cu} 1-$ in the $8 i(0,0.5, z), \mathrm{Cu} 2$ - in the $8 h(0,0, z), \mathrm{Si} 1$ - in the $8 g(0, y, 1 / 4), \mathrm{Si} 2$ in the $8 f(x, 0,1 / 4), \mathrm{Si} 3, \mathrm{Si} 4, \mathrm{O} 6$, and 07 -in the $8 j(x, y, 0)$ sites correspondingly, all the others-in the general $16 k$ positions $(x, y, z)$. All positional parameters were refined without any constraints.

\begin{tabular}{|c|c|c|c|c|}
\hline \multicolumn{5}{|c|}{$\mathrm{BaCuSi}_{2} \mathrm{O}_{6}$ Lattice Parameters at $T=13 \mathrm{~K}$} \\
\hline$a, \AA$ & \multicolumn{4}{|c|}{$9.95129(7)$} \\
\hline$b, \AA$ & \multicolumn{4}{|c|}{$9.96792(7)$} \\
\hline$c, \AA$ & \multicolumn{4}{|c|}{$22.23914(13)$} \\
\hline $\mathrm{V}, \AA^{3}$ & \multicolumn{4}{|c|}{$2205.98(3)$} \\
\hline Atom & $x$ & $y$ & $z$ & $B_{\text {iso }}, \AA^{2}$ \\
\hline $\mathrm{Ba}$ & $0.2567(5)$ & $0.2444(5)$ & $0.1234(3)$ & $0.09(5)$ \\
\hline $\mathrm{Cu} 1$ & 0 & 0.5 & $0.0624(2)$ & $0.60(3)$ \\
\hline $\mathrm{Cu} 2$ & 0 & 0 & $0.1893(2)$ & $0.60(3)$ \\
\hline Si1 & 0 & $0.2761(11)$ & 0.25 & $0.29(4)$ \\
\hline $\mathrm{Si} 2$ & $0.2773(12)$ & 0 & 0.25 & $0.29(4)$ \\
\hline $\mathrm{Si} 3$ & $0.0019(9)$ & $0.7724(11)$ & 0 & $0.29(4)$ \\
\hline $\mathrm{Si} 4$ & $0.7771(13)$ & $0.0067(9)$ & 0 & $0.29(4)$ \\
\hline $\mathrm{O} 1$ & $0.0362(5)$ & $0.8095(5)$ & $0.3089(3)$ & $0.77(3)$ \\
\hline $\mathrm{O} 2$ & $0.3054(6)$ & $0.4622(5)$ & $0.1891(3)$ & $0.77(3)$ \\
\hline $\mathrm{O} 3$ & $0.3076(5)$ & $0.0092(5)$ & $0.0608(3)$ & $0.77(3)$ \\
\hline $\mathrm{O} 4$ & $0.5123(5)$ & $0.1918(5)$ & $0.0626(4)$ & $0.77(3)$ \\
\hline O5 & $0.1281(6)$ & $0.3730(5)$ & $0.2698(2)$ & $1.09(4)$ \\
\hline O6 & $0.3454(7)$ & $0.3454(7)$ & 0 & $1.09(4)$ \\
\hline $\mathrm{O} 7$ & $0.1042(7)$ & $0.1043(7)$ & 0 & $1.09(4)$ \\
\hline
\end{tabular}

$I 4_{1} /$ acd, gives the first sensible solution. Yet the next group Ibam (No. 72) supplies a definitely better model. Compared to the Ibca model, the model in Ibam provides a significant improvement of the agreement facttors: $\chi^{2}$ decreases from 4.33 to 2.66 , and the Bragg $R$-factor-from 7.05 to 4.80 . Both structure models are in fact slight distortions of the RT tetragonal structure with $I 4_{1} /$ acd symmetry, but are having very essential difference between each other from the point of view of the geometry of the $\mathrm{Cu}-\mathrm{Cu}$ dimer layers and their stacking along the direction perpendicular to the $\mathrm{Cu}_{2}\left(\mathrm{SiO}_{3}\right)_{4}$ layers. The unique $\mathrm{Cu}$ atom in the $\mathrm{RT}$ structure model is being split into two distinct atoms in both models, but in the case of the $I b c a$ model they are forming just one type of $\mathrm{Cu}-\mathrm{Cu}$ dimer layer, while the correct Ibam model contains two distinctly different $\mathrm{Cu}-\mathrm{Cu}$ dimer layers, and these will be discussed below. The Rietveld refinement plot obtained with the neutron data is shown in Fig. 4. The refined parameters of the LT crystal structure are given in Table I, and the most relevant interatomic distances in Table II. The schematic representation of the crystal structure of the LT phase of $\mathrm{BaCuSi}_{2} \mathrm{O}_{6}$ is given in Fig. 5.

Unlike in the single crystal experiments reported in Ref. 24, our study carried out by powder diffraction could not reveal
TABLE II. The most relevant interatomic distances $(\AA)$ in the crystal structure of the LT phase of $\mathrm{BaCuSi}_{2} \mathrm{O}_{6}$ refined from the neutron powder diffraction data at $T=13 \mathrm{~K}$.

\begin{tabular}{lc}
\hline \hline $\mathrm{Cu} 1-\mathrm{Cu} 1$ & $2.774(6)$ \\
$\mathrm{Cu} 2-\mathrm{Cu} 2$ & $2.701(6)$ \\
$\mathrm{Cu} 1-\mathrm{Cu} 2$ & $5.728(3)$ and $5.720(2)$ \\
$\langle\mathrm{Si} 1-\mathrm{O}(1,1,5,5)\rangle$ & $1.632(16)$ \\
$\langle\mathrm{Si} 2-\mathrm{O}(2,2,5,5)\rangle$ & $1.634(18)$ \\
$\langle\mathrm{Si} 3-\mathrm{O}(4,4,6,7)\rangle$ & $1.63(2)$ \\
$\langle\mathrm{Si} 4-\mathrm{O}(3,3,6,7)\rangle$ & $1.61(2)$ \\
\hline \hline
\end{tabular}

the presence of any incommensurate peaks due to their relative weakness: According to Ref. 24, the central Bragg peaks did have to be scaled down by a factor of $\sim 10^{4}$ to display on the same scale with the satellite incommensurate modulation peaks. Such dynamic ranges are inaccessible for powder diffraction, and in this sense, the presence of the incommensurate modulation did not hinder the determination of what we believe to be a most probable model for the average LT crystal structure of $\mathrm{BaCuSi}_{2} \mathrm{O}_{6}$.

An interesting feature is observed in the geometry of the $\mathrm{Cu}_{2}\left(\mathrm{SiO}_{3}\right)_{4}$ layers which is very relevant for the interpretation of low-temperature magnetic properties: Mutual rotation of the $\mathrm{CuO}_{4}$ coordination polyhedra in the $\mathrm{Cu}$-dimer layers, characteristic for the RT structure on $\mathrm{BaCuSi}_{2} \mathrm{O}_{6}$, is only preserved in the layers with shorter $\mathrm{Cu}-\mathrm{Cu}$ dumbbell distances. This feature is illustrated in Fig. 6: At $130 \mathrm{~K}$, in the tetragonal phase, all the $\mathrm{Cu}-\mathrm{Cu}$ dimer layers are equivalent and the $\mathrm{CuO}_{4}$ coordination polyhedra look like squares slightly turned $\left(\sim 19^{\circ}\right)$ with respect to each other in each $\mathrm{Cu}-\mathrm{Cu}$ dumbbell around its axis [Fig. 6(a): view along the $c$ direction of the unit cell]. At low temperature, only the $\mathrm{Cu} 2-\mathrm{Cu} 2$ dimer
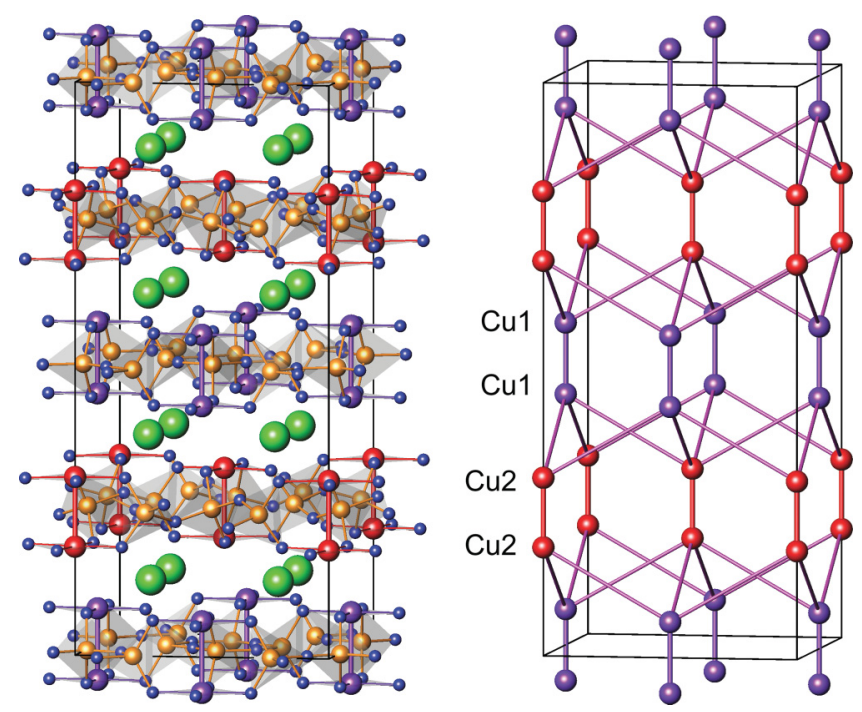

FIG. 5. (Color online) Schematic representation of the crystal structure of the LT phase of $\mathrm{BaCuSi}_{2} \mathrm{O}_{6}$. In the right panel, only the $\mathrm{Cu}-\mathrm{Cu}$ dimers are shown. The $\mathrm{Cu} 1-\mathrm{Cu} 1$ distance of 2.774(6) $\AA$ is much longer than the $\mathrm{Cu} 2-\mathrm{Cu} 2$ with 2.701(6) $\AA$. For the interlayer coupling paths, $\mathrm{Cu} 1-\mathrm{Cu} 2$ (shown in thin lines in the right figure), there are always two pairs of slightly inequivalent bond distances (see Table II). 

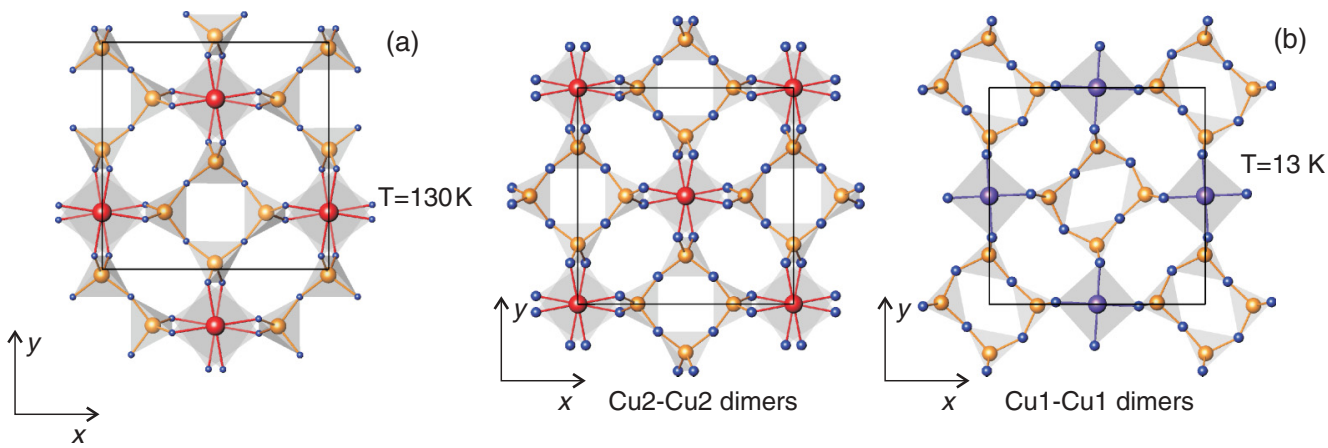

FIG. 6. (Color online) $\mathrm{Cu}-\mathrm{Cu}$ dimer layer structure. (a) In tetragonal phase (the figure is based on the atom positions refined from the neutron data at $130 \mathrm{~K})$. $\mathrm{Cu}-\mathrm{Cu}$ distance is 2.731(2) $\AA$. (b) Two types of $\mathrm{Cu}-\mathrm{Cu}$ dimer layers at low temperature as refined from the neutron data taken at $13 \mathrm{~K}$. The $\mathrm{Cu} 2-\mathrm{Cu} 2$ distance is 2.701(6) $\AA$; the $\mathrm{Cu} 1-\mathrm{Cu} 1$ distance is 2.774(6) $\AA$.

layer with shorter $\mathrm{Cu}-\mathrm{Cu}$ distances, shown on the left of Fig. 6(b), preserves this feature (the mutual rotation angles of the $\mathrm{Cu}_{2} \mathrm{O}_{4}$ squares with respect to each other are on the order of $\sim 21^{\circ} \ldots 22^{\circ}$ ), and the $\mathrm{Si}_{4} \mathrm{O}_{12}$ rings in these layers are also (same as at $130 \mathrm{~K}$ ) nearly squares. In contrast to this, in the $\mathrm{Cu} 1-\mathrm{Cu} 1$ dimer layer with longer $\mathrm{Cu}-\mathrm{Cu}$ distances, shown on the right of Fig. 6(b), the $\mathrm{CuO}_{4}$ coordination polyhedra are not turned with respect to each other. The $\mathrm{Si}_{4} \mathrm{O}_{12} 4$-member rings in the $\mathrm{Cu} 1-\mathrm{Cu} 1$ dimer layers show clear deviations from the ideal square shape, forming a checkerboard type of ordering of their elongation directions along one of the $a-b$ plane diagonals. The intralayer $\mathrm{Cu}-\mathrm{Cu}$ distances are identical in both types of layers in our model, yet it is too hard to quantify the obvious differences in the exact intralayer exchange paths between the dimers, which are obviously nonequivalent for the layers with the shorter and the longer $\mathrm{Cu}-\mathrm{Cu}$ dimers.

\section{B. First-order character of the transition}

In agreement with the results already reported in Ref. 24, the structural transition we observe in $\mathrm{BaCuSi}_{2} \mathrm{O}_{6}$ is of first order. The refined unit cell volumes, as well as the weight proportions of the tetragonal and orthorhombic phases on cooling and on heating, are shown in Fig. 7. The observed discontinuity in the unit cell volume at the transition is $\sim 1 \%$. The $50: 50$ proportions of the phases are reached at $\sim 89.7 \mathrm{~K}$ on cooling and at $\sim 107.2 \mathrm{~K}$ on heating. The width of the hysteresis is thus $\sim 17.5 \mathrm{~K}$.

\section{Possible alternative structure models}

Dealing with the neutron powder data set with limited intensity does not in principle exclude the possibility of having missed the lowering of the symmetry just due to the fact that the very weak diffraction intensities may potentially be confused with systematic absences. Having carried out an exhaustive search in the orthorhombic syngony for alternative crystal structure models of the LT phase of $\mathrm{BaCuSi}_{2} \mathrm{O}_{6}$, and upon carrying out careful refinements of their parameters, we can as well list all other possible structure models providing comparable agreement factors. It turns out that all alternative possibilities which deserve being considered are limited to the subgroups of the space group I bam.
In Table III, we have summarized all hypothetically possible models for the structure of the LT phase of $\mathrm{BaCuSi}_{2} \mathrm{O}_{6}$ providing plausible refinements, along with the key
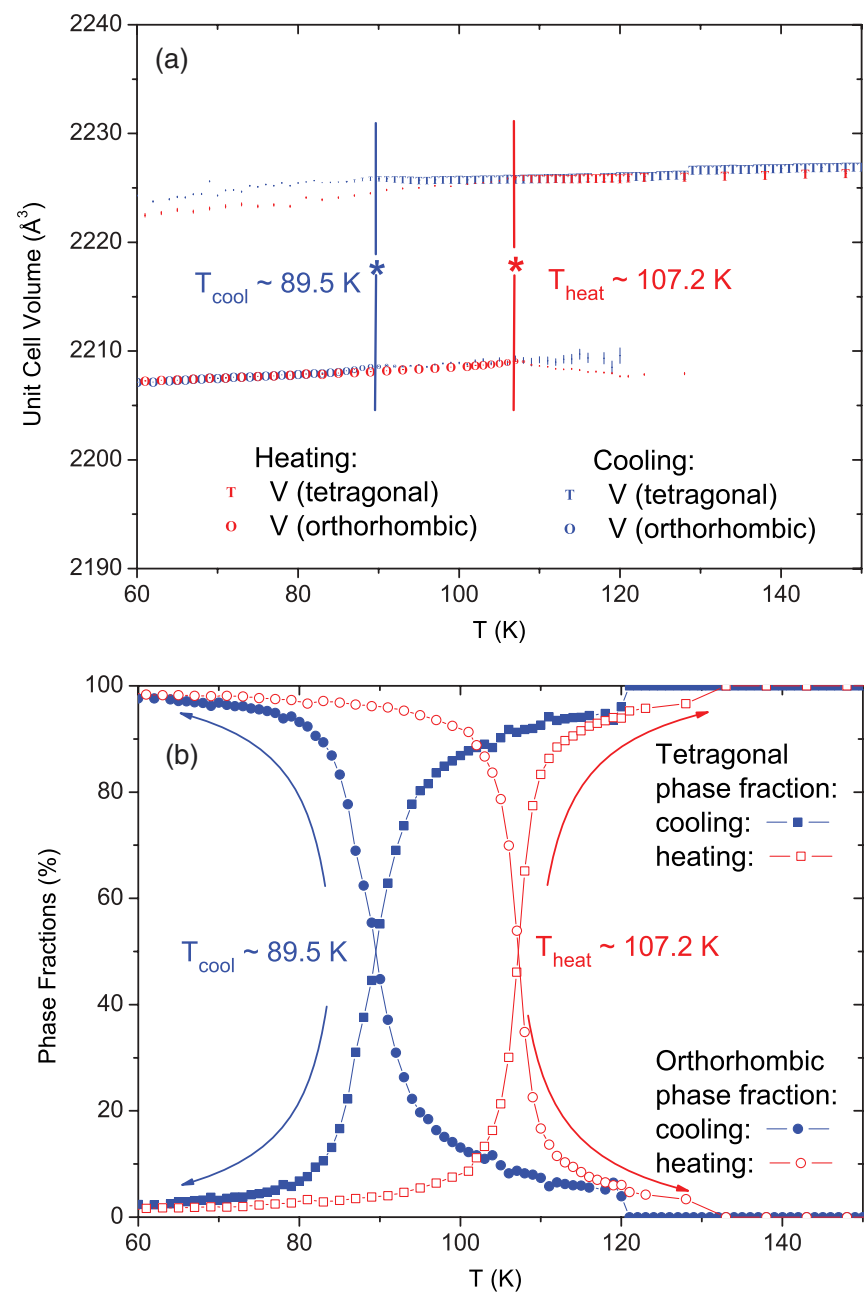

FIG. 7. (Color online) Unit cell volumes on cooling and on heating (a) and the relative phase weight proportions (b) for the tetragonal and orthorhombic modifications of $\mathrm{BaCuSi}_{2} \mathrm{O}_{6}$. Results of the refinements done with the synchrotron $\mathrm{x}$-ray data. Symbol sizes $(\mathrm{T})$ and $(\mathrm{O})$ in panel (a) are proportional to the corresponding weight phase fractions. Error bars in (a) are also shown and are typically much smaller than the symbol sizes. 
TABLE III. List of all possible space groups for the crystal structure of the LT phase of $\mathrm{BaCuSi}_{2} \mathrm{O}_{6}$. SG and Set denote the space group number and particular setting. $N_{a}$ and $N_{p}$ are the total numbers of independent atoms and of refined positional parameters (atomic coordinates). $N_{\mathrm{Cu}}$ and $N_{D}$ denote the numbers of independent $\mathrm{Cu}$ atoms and of the unique $\mathrm{Cu}-\mathrm{Cu}$ dimer layers in each model correspondingly. $\mathrm{Cu}-\mathrm{Cu}$ distances for different dimer layers are separated by slashes. The agreement factors $\chi^{2}, R_{p}$ correspond to the refinements based on neutron powder diffraction data collected at $T=13 \mathrm{~K}$.

\begin{tabular}{|c|c|c|c|c|c|}
\hline SG & Set & $N_{a}, N_{p}$ & $N_{\mathrm{Cu}}, N_{D}$ & $\mathrm{Cu}-\mathrm{Cu}, \AA$ & $\chi^{2}, R_{p}$ \\
\hline 72 & Ibam & 14,30 & 2,2 & $2.701(6) / 2.774(6)$ & $2.66,4.80$ \\
\hline 60 & $P b c n^{\mathrm{a}}$ & 21,59 & 2,2 & $2.713(6) / 2.768(6)$ & $2.32,4.41$ \\
\hline 57 & $\mathrm{Pbcm}^{\mathrm{a}}$ & 25,63 & 2,2 & $2.705(6) / 2.770(6)$ & $2.55,4.69$ \\
\hline 56 & $P c c n^{\mathrm{a}}$ & 22,58 & 4,2 & $2.71(2) / 2.78(3)$ & $2.42,4.53$ \\
\hline 55 & $P_{b a m^{\mathrm{a}}}$ & 26,62 & 4,4 & $\begin{array}{c}2.71(2) / 2.70(2) / \\
2.86(2) / 2.70(2)\end{array}$ & $2.59,4.75$ \\
\hline 50 & $P_{b a n^{\mathrm{a}}}$ & 24,56 & 4,4 & $\begin{array}{c}2.71(2) / 2.77(3) / \\
2.70(2) / 2.77(3)\end{array}$ & $2.36,4.45$ \\
\hline 49 & $P c c m^{\mathrm{a}}$ & 28,60 & $4,2^{\mathrm{b}}$ & $\begin{array}{l}2.73(3) \text { and } 2.82(2) / \\
2.69(3) \text { and } 2.72(3)\end{array}$ & $2.46,4.59$ \\
\hline 46 & $I 2 \mathrm{~cm}^{\mathrm{a}}$ & 25,62 & 2,2 & $2.705(6) / 2.767(6)$ & $2.50,4.61$ \\
\hline 45 & $\operatorname{Iba} 2^{\mathrm{a}}$ & 22,57 & 4,2 & $2.71(3) / 2.78(3)$ & $2.36,4.46$ \\
\hline 33 & $P 2{ }_{1} c n^{\mathrm{c}}$ & 40,119 & 4,2 & $2.71(3) / 2.77$ (4) & $2.26,4.32$ \\
\hline 32 & $P b a 2^{c}$ & 44,115 & 8,4 & $\begin{array}{c}2.78(6) / 2.76(8) / \\
2.67(8) / 2.80(8)\end{array}$ & $2.23,4.31$ \\
\hline 30 & $P 2 a n^{c}$ & 42,117 & 4,4 & $\begin{array}{c}2.73(3) / 2.77(3) / \\
2.68(3) / 2.77(3)\end{array}$ & $2.19,4.23$ \\
\hline 29 & $P \mathrm{Ca} 2_{1}{ }^{\mathrm{c}}$ & 40,119 & 4,2 & $2.74(6) / 2.76(6)$ & $2.12,4.17$ \\
\hline 28 & $P 2 \mathrm{~cm}^{\mathrm{c}}$ & 50,125 & $4,2^{b}$ & $\begin{array}{l}2.67(4) \text { and } 2.74(4) / \\
2.76(5) \text { and } 2.78(5)\end{array}$ & $2.29,4.34$ \\
\hline 27 & $P c c 2^{c}$ & 44,115 & $8,2^{\mathrm{b}}$ & $\begin{array}{c}2.76(9) \text { and } 2.78(9) / \\
2.76(6) \text { and } 2.69(6)\end{array}$ & $2.14,4.20$ \\
\hline 26 & $P 2_{1} a m^{c}$ & 48,127 & 4,4 & $\begin{array}{c}2.69(3) / 2.70(3) / \\
2.86(2) / 2.70(3)\end{array}$ & $2.36,4.42$ \\
\hline 23 & $I 222^{\mathrm{a}}$ & 24,56 & $4,2^{b}$ & $\begin{array}{c}2.70(3) \text { and } 2.71(3) / \\
2.75(3)\end{array}$ & $2.43,4.51$ \\
\hline 18 & $P 22_{1} 2_{1}{ }^{\mathrm{c}}$ & 42,118 & $4,2^{\mathrm{b}}$ & $\begin{array}{c}2.67(3) \text { and } 2.74(3) / \\
2.77(5)\end{array}$ & $2.23,4.28$ \\
\hline 16 & $P 222$ & 48,112 & $8,4^{\mathrm{b}}$ & $\begin{array}{l}2.85(4) \text { and } 2.70(4) / \\
2.78(6) \text { and } 2.75(6) / \\
2.68(4) \text { and } 2.62(5) / \\
2.78(6) \text { and } 2.75(6)\end{array}$ & $2.22,4.25$ \\
\hline
\end{tabular}

a Subgroup of space group 72 (Ibam).

${ }^{b}$ Different $\mathrm{Cu}-\mathrm{Cu}$ distances within one or more dimer layers.

${ }^{\mathrm{c}}$ Subgroup of a few space groups which are subgroups of space group 72 (I bam).

values - the numbers of the independent unique $\mathrm{Cu}-\mathrm{Cu}$ dimer layers, the lengths of $\mathrm{Cu}-\mathrm{Cu}$ dimers, some statistical indicators for the complexities of the models, and the corresponding agreement factors of the refinements. We find it inappropriate to present here all the structure models in details. The groupsubgroup transformations are straightforward, and any models will be supplied on request. The general trend is that all alternative possibilities provide a pattern of the $\mathrm{Cu}-\mathrm{Cu}$ dimer layers either very similar to or even more complex than that in our model with the space group Ibam (Tables I and II). For some of them we have not 2 but 4 independent $\mathrm{Cu}-\mathrm{Cu}$ dimer layers. Since the apparent improvement in agreement factors of the refinements for lower symmetries is only achieved when the number of independent atoms and refinable parameters is getting very high, and due to the obvious similarity to the highest symmetry model with the space group Ibam (Tables I and II), we strongly tend to believe that the true structure of the
LT phase of $\mathrm{BaCuSi}_{2} \mathrm{O}_{6}$ may sufficiently well and adequately be described by our model with the space group I bam. Some later studies with higher precision may one day show slight deviations from this model to one of those listed in Table III.

\section{DISCUSSION AND SUMMARY}

We have investigated the low-temperature structural phase transition in $\mathrm{BaCuSi}_{2} \mathrm{O}_{6}$ by means of powder synchrotron $\mathrm{x}$-ray and neutron diffraction and have determined the most probable average crystal structure of its LT phase. The proposed crystal structure model of the LT phase of $\mathrm{BaCuSi}_{2} \mathrm{O}_{6}$ has space group $\mathrm{Ibam}$, and contains two individual $\mathrm{Cu}$ atom positions (with only one in the room-temperature structure). These two $\mathrm{Cu}$ atoms are forming two types of $\mathrm{Cu}-\mathrm{Cu}$ dimers thus altering the most essential magnetic $\mathrm{Cu}-\mathrm{Cu}$ exchange path lengths in a stacking manner along the $c$ axis. The 
interlayer $\mathrm{Cu}-\mathrm{Cu}$ exchange distances are also becoming barely different at low temperature, forming in total two distinct but close interlayer $\mathrm{Cu}-\mathrm{Cu}$ distances. In agreement with the previous study (Ref. 24), we confirm the phase transition to be of first order, with a unit cell volume discontinuity of $\sim 1 \%$ at the transition temperature, and a temperature hysteresis of $\sim 17.5 \mathrm{~K}$ (the $50: 50$ phase weight proportions being achieved at $\sim 89.7 \mathrm{~K}$ on cooling and at $\sim 107.2 \mathrm{~K}$ on heating). In the LT crystal structure, the layers with shorter $\mathrm{Cu}-\mathrm{Cu}$ dimer distances $(\sim 2.701 \AA$ at $13 \mathrm{~K})$ preserve the mutual rotation of the $\mathrm{CuO}_{4}$ coordination squares with respect to each other in the $\mathrm{Cu}-\mathrm{Cu}$ dumbbells and the squarelike shapes of the $\left(\mathrm{SiO}_{3}\right)_{4}$ molecules around them. This feature which is characteristic for the room-temperature structure is lost in the layers with longer $\mathrm{Cu}-\mathrm{Cu}$ bonds $(\sim 2.774 \AA$ at $13 \mathrm{~K}$ ); the coordination $\mathrm{CuO}_{4}$ squares in them unfold the mutual rotation and are aligned identically, and the $\left(\mathrm{SiO}_{3}\right)_{4}$ molecules get strongly elongated along one of the $a-b$ diagonals, forming a checkerboard pattern of their elongation directions. Thus, in the LT crystal structure of $\mathrm{BaCuSi}_{2} \mathrm{O}_{6}$, the two distinct adjacent $\mathrm{Cu}_{2}\left(\mathrm{SiO}_{3}\right)_{4}$ layers with different $\mathrm{Cu}-\mathrm{Cu}$ dimer distances and even different internal geometries are alternating in a sequential manner along the $c$ axis. This finding corroborates the interpretation of the low-temperature INS data ${ }^{5}$ in terms of existence of two strongly inequivalent dimer layers in $\mathrm{BaCuSi}_{2} \mathrm{O}_{6}$ with possible further complex modifications to one of them. The lower energy excitations reported in Ref. 5 would correspond to the $\mathrm{Cu} 2-\mathrm{Cu} 2$ dimer layers with shorter $\mathrm{Cu}-\mathrm{Cu}$ distances, while the higher energy multimode excitations correspond to the $\mathrm{Cu} 1-\mathrm{Cu} 1$ dimer layers with longer $\mathrm{Cu}-\mathrm{Cu}$ distances. The incommensurate modulation of the crystal structure observed before in different studies did not hinder the structure determination, since as shown in Ref. 24 the intensities of the satellite reflections are $\sim 10^{4}$ times weaker than that of the fundamental reflections, and thus they did not disturb our powder diffraction based average structure determination.
Here we want to conclude with a remark about the possibility of Dzyaloshinskii-Moriya (DM) interactions for $\mathrm{BaCuSi}_{2} \mathrm{O}_{6}$. All three, HT, RT, and LT, structures of $\mathrm{BaCuSi}_{2} \mathrm{O}_{6}$ are centrosymmetric. However, at RT there is no inversion center in the middle of the $\mathrm{Cu}-\mathrm{Cu}$ dimers, as correctly pointed out by Ref. 24. The same holds for LT $\mathrm{Cu} 2-\mathrm{Cu} 2$ dimer layers with shorter $\mathrm{Cu}-\mathrm{Cu}$ distances. In contrast to this, the LT $\mathrm{Cu} 1-\mathrm{Cu} 1$ dimer layer with longer $\mathrm{Cu}-\mathrm{Cu}$ distances has similarly to the HT $I 4 / \mathrm{mmm}$ structure the inversion symmetry in the middle of the $\mathrm{Cu}$ dimer and the intradimer DM coupling would be forbidden. In the real structure of $\mathrm{BaCuSi}_{2} \mathrm{O}_{6}$, the incommensurate modulation may well remove this local symmetry and make DM interaction allowed again.

We note that the results presented here provide a structural model for the compound which justifies the interpretation of previous INS and NMR data by a number of groups ${ }^{5,19}$ in terms of two inequivalent dimer layers in $\mathrm{BaCuSi}_{2} \mathrm{O}_{6}$ with further complex modifications to one of the layers that may partially relieve frustration. Furthermore, the detailed structure information provided here will be important input for the ongoing $a b$ initio calculations of the exchange interactions in this fascinating material.

\section{ACKNOWLEDGMENTS}

This work is based on the experiments carried out at the Swiss Light Source synchrotron and at the Swiss Spallation Neutron Source SINQ, Paul Scherrer Institute, Villigen, Switzerland. We acknowledge discussions with C. Berthier, M. Horvatić, S. Krämer, E. Joon, J. Mesot, C. Batista, F. Mila, A. Tsirlin, F. Duc, and K. Sparta. Our special thanks go to C. Rüegg for numerous inspiring discussions and his careful reading of the manuscript. Work in Tallinn was supported by the Estonian Ministry of Education and Research under Grants No. SF0690029s09 and No. SF0690034s09, and Estonian Science Foundation under Grants No. ETF8198 and No. ETF8440.

\footnotetext{
*denis.cheptiakov@psi.ch

${ }^{\dagger}$ raivo.stern@kbfi.ee

${ }^{1}$ I. Affleck, Phys. Rev. B 43, 3215 (1991).

${ }^{2}$ T. Giamarchi and A. M. Tsvelik, Phys. Rev. B 59, 11398 (1999).

${ }^{3}$ S. Sachdev, Quantum Phase Transitions (Cambridge University Press, Cambridge, 1999), p. 353.

${ }^{4}$ T. M. Rice, Science 298, 760 (2002).

${ }^{5}$ C. Rüegg, D. F. McMorrow, B. Normand, H. M. Ronnow, S. E. Sebastian, I. R. Fisher, C. D. Batista, S. N. Gvasaliya, C. Niedermayer, and J. Stahn, Phys. Rev. Lett. 98, 017202 (2007).

${ }^{6}$ T. Giamarchi, C. Rüegg, and O. Tchernyshyov, Nat. Phys. 4, 198 (2008).

${ }^{7}$ C. Rüegg, N. Cavadini, A. Furrer, H. U. Gudel, K. Kramer,

H. Mutka, A. K. Habicht, P. Vorderwisch, and A. Wildes, Nature (London) 423, 62 (2003).

${ }^{8}$ H. Kageyama, K. Yoshimura, R. Stern, N. V. Mushnikov, K. Onizuka, M. Kato, K. Kosuge, C. P. Slichter, T. Goto, and Y. Ueda, Phys. Rev. Lett. 82, 3168 (1999).
}

${ }^{9}$ K. Kodama, M. Takigawa, M. Horvatić, C. Berthier, H. Kageyama, Y. Ueda, S. Miyahara, F. Becca, and F. Mila, Science 298, 395 (2002).

${ }^{10}$ M. Jaime, V. F. Correa, N. Harrison, C. D. Batista, N. Kawashima, Y. Kazuma, G. A. Jorge, R. Stern, I. Heinmaa, S. A. Zvyagin, Y. Sasago, and K. Uchinokura, Phys. Rev. Lett. 93, 087203 (2004).

${ }^{11}$ S. E. Sebastian, N. Harrison, C. D. Batista, L. Balicas, M. Jaime, P. A. Sharma, N. Kawashima, and I. R. Fisher, Nature (London) 441, 617 (2006).

${ }^{12}$ K. M. Sparta and G. Roth, Acta Crystallogr. Sect. B 60, 491 (2004).

${ }^{13}$ L. W. Finger, R. M. Hazen, and R. J. Hemley, Am. Mineral. 74, 952 (1989).

${ }^{14}$ Y. Sasago, K. Uchinokura, A. Zheludev, and G. Shirane, Phys. Rev. B 55, 8357 (1997).

${ }^{15}$ S. A. Zvyagin, J. Wosnitza, J. Krzystek, R. Stern, M. Jaime, Y. Sasago, and K. Uchinokura, Phys. Rev. B 73, 094446 (2006).

${ }^{16}$ C. D. Batista, J. Schmalian, N. Kawashima, P. Sengupta, S. E. Sebastian, N. Harrison, M. Jaime, and I. R. Fisher, Phys. Rev. Lett. 98, 257201 (2007). 
${ }^{17}$ J. Schmalian and C. D. Batista, Phys. Rev. B 77, 094406 (2008).

${ }^{18}$ M. Horvatić, C. Berthier, F. Tedoldi, A. Comment, M. Sofin, M. Jansen, and R. Stern, Prog. Theor. Phys. Suppl. 159, 106 (2005).

${ }^{19}$ S. Krämer, R. Stern, M. Horvatić, C. Berthier, T. Kimura, and I. R. Fisher, Phys. Rev. B 76, 100406 (2007).

${ }^{20}$ O. Rösch and M. Vojta, Phys. Rev. B 76, 180401 (2007).

${ }^{21}$ O. Rösch and M. Vojta, Phys. Rev. B 76, 224408 (2007).

${ }^{22}$ N. Laflorencie and F. Mila, Phys. Rev. Lett. 102, 060602 (2009).

${ }^{23}$ S. E. Sebastian, P. A. Sharma, M. Jaime, N. Harrison, V. Correa, L. Balicas, N. Kawashima, C. Batista, and I. R. Fisher, Phys. Rev. B 72, 100404 (2005).
${ }^{24}$ E. C. Samulon, Z. Islam, S. E. Sebastian, P. B. Brooks, M. K. McCourt, J. Ilavsky, and I. R. Fisher, Phys. Rev. B 73, 100407 (2006).

${ }^{25}$ http://www.psi.ch/sls/ms.

${ }^{26}$ P. Fischer, G. Frey, M. Koch, M. Könnecke, V. Pomjakushin, J. Schefer, R. Thut, N. Schlumpf, R. Bürge, U. Greuter, S. Bondt, and E. Berruyer, Physica B 276, 146 (2000).

${ }^{27}$ V. Favre-Nicolin and R. Černý, J. Appl. Crystallogr. 35, 734 (2002).

${ }^{28}$ J. Rodríguez-Carvajal, Physica B 192, 55 (1993). 